\title{
Nerve ultrasound for differentiation between amyotrophic lateral sclerosis and multifocal motor neuropathy
}

\author{
Alexander Grimm • Bernhard F. Décard • \\ Ioanna Athanasopoulou • Kathi Schweikert • \\ Michael Sinnreich $\cdot$ Hubertus Axer
}

Received: 25 November 2014/Revised: 12 January 2015/Accepted: 13 January 2015/Published online: 28 January 2015

(c) Springer-Verlag Berlin Heidelberg 2015

\begin{abstract}
Ultrasound is useful for non-invasive visualization of focal nerve pathologies probably resulting from demyelination, remyelination, edema or inflammation. In patients with progressive muscle weakness, differentiation between multifocal motor neuropathy (MMN) and amyotrophic lateral sclerosis (ALS) is essential regarding therapy and prognosis. Therefore, the objective of this study was to investigate whether nerve ultrasound can differentiate between ALS and MMN. Systematic ultrasound measurements of peripheral nerves and the 6th cervical nerve root (C6) were performed in 17 patients with ALS, in 8 patients with MMN and in 28 healthy controls. Nerve conduction studies of corresponding nerves were undertaken in MMN and ALS patients. Electromyography was performed in ALS patients according to revised El-Escorial criteria. ANOVA and unpaired $t$ test with Bonferroni correction revealed significant differences in cross-sectional areas (CSA) of different nerves and C6 diameter between the groups. Nerve enlargement was found significantly more frequently in MMN than in other groups $(p<0.001)$. Receiver operating characteristics analysis revealed detection of enlarged nerves/roots in at least four
\end{abstract}

A. Grimm $(\varangle)$ · B. F. Décard · I. Athanasopoulou •

K. Schweikert · M. Sinnreich

Department of Neurology, Neuromuscular Center, Basel

University Hospital, University Basel, Petersgraben 4,

4000 Basel, Switzerland

e-mail: alexander.grimm@usb.ch

H. Axer

Hans Berger Department of Neurology, Jena University

Hospital, Jena, Germany

H. Axer

Center for Sepsis Control and Care (CSCC), Jena University

Hospital, Jena, Germany measurement points to serve as a good marker to differentiate MMN from ALS with a sensitivity of $87.5 \%$ and a specificity of $94.1 \%$. Ultrasonic focal nerve enlargement in MMN was often not colocalized with areas of conduction blocks found in nerve conduction studies. Systematic ultrasound measurements in different nerves and nerve roots are valuable for detecting focal nerve enlargement in MMN, generally not found in ALS and thus could serve as a diagnostic marker to differentiate between both entities in addition to electrodiagnostic studies.

Keywords Nerve ultrasound - MMN - Polyneuropathy · Cross-sectional area $\cdot$ ALS
Abbreviations
ALS Amyotrophic lateral sclerosis
AUC Area under the curve
CIDP Chronic inflammatory demyelinating
polyradiculoneuropathy
CMAP Compound muscle action potential
CMT Charcot Marie Tooth
CSA Cross-sectional area
CV Conduction velocity
C6 Cervical nerve root 6
EFNS European Federation of Neurological Societies/
Peripheral Nerve Society
GBS Guillain-Barré syndrome
ICC Intraclass correlation coefficient
ICSAV Intranerve CSA variability
LMN Lower motor neuron
MMF Mycophenolatmofetil
MMN Multifocal motor neuropathy
NCS Nerve conduction studies
PNP Polyneuropathy
PNUS Peripheral nerve ultrasound 
PSW Positive sharp waves

ROC Receiver operating characteristics

SNAP Sensory nerve action potential

UMN Upper motor neuron

\section{Introduction}

Amyotrophic lateral sclerosis (ALS), a fatal neuromuscular disorder affecting the upper and lower motoneurons (UMN and LMN) leads to progressive muscle weakness, atrophy, and bulbar symptoms [1, 2]. Deep tendon reflexes are mostly exaggerated. Death usually occurs 2-3 years following diagnosis due to complications related to immobility and respiratory failure. Diagnosis of ALS may be delayed due to lack of biomarkers [3]. However, diagnosis of ALS can be supported by electromyographic findings such as fibrillations, positive sharp waves (PSW) or fasciculations as a sign of LMN involvement and pathologic motor-evoked potentials with delayed central motor latency as an electrophysiological correlate of pyramidal tract involvement [4, 5]. However, fibrillations, PSW, and fasciculations can also be apparent in other neurogenic disorders, e.g. polyneuropathies, radiculopathies, and other peripheral nerve lesions.

Muscle ultrasound can facilitate and accelerate diagnosis of ALS by increasing the field of view for detecting fasciculations at a higher sensitivity in bulbar muscles, e.g. the tongue and thoracic muscles [6]. However, in some instances, differentiation from other disorders may be difficult, in particular multifocal motor neuropathy (MMN).

Although MMN is rare, differentiation from ALS is essential with regard to treatment and prognosis. MMN is a treatable polyneuropathy affecting peripheral motor nerves, often following the distribution of individual nerves without involvement of sensory nerve fibers [7, 8]. Disease course can be stepwise or chronic progressive and although deep tendon reflexes are mostly reduced or absent, these may also be preserved. MMN diagnosis is based on high titres of anti-ganglioside(G)M1-immunoglobulin $\mathrm{M}$ antibodies and finding of (multifocal) conduction blocks outside of common nerve entrapment sites $[9,10]$ without signs of UMN involvement. A conduction block is the failure of transmission of an electrical impulse in a circumscribed region along a peripheral nerve, and is mostly due to focal demyelination. It has been suggested that the disease is possibly immunologically mediated by binding of anti-GM1 antibodies to neural structures [10]. In some instances, diagnosis of $\mathrm{MMN}$ is more challenging in cases where conduction blocks cannot be detected.
The role of peripheral nerve ultrasound (PNUS) in detecting nerve enlargement and changes in echogenicity such as hypoechoic fascicles, hyperechoic epineurium or increased vascularization in inherited and immune-mediated neuropathies have been previously described [11-23]. Reports indicate that PNUS can differentiate between acquired immune-mediated demyelinating neuropathies and axonal neuropathies by demonstrating nerve enlargement in patients with acquired demyelinating diseases, since axonal types of polyneuropathy do not regularly show nerve enlargement [11]. Similar findings have also been described for inherited demyelinating and axonal CharcotMarie-Tooth disease (CMT) [21]. In inherited neuropathies, generalized nerve enlargement occurs particularly in CMT 1A, whereas in immune-mediated neuropathies, different patterns of nerve alterations ranging from normal to focal as well as generalized alterations are seen [18, 21]. In vasculitic neuropathies with axonal pattern of damage, focal nerve enlargement also occurs [22, 23]. Thus, it has to be hypothesized that nerve enlargement, altered echointensity and vascularization could not only represent an expression of de- and remyelination, but also signify a sign of focal inflammation, edema, epineural fibrosis, and focal damage in axonal as well as in demyelinating inflammatory neuropathies [17, 18, 23].

Accordingly, description of focal nerve enlargement in MMN patients by a number of authors is not surprising [20, 24, 25]. In ALS, nerve enlargement has, however, never been detected and the authors describe a normal or even reduced cross-sectional area (CSA) of upper extremity nerves and a reduced diameter of the cervical nerve roots [26-28].

The aim of this study was firstly, to compare results of CSA measurements in nerves of upper and lower extremities, vagus nerve, and the sixths cervical nerve root in both ALS and MMN and, secondly, to determine the role of PNUS in differentiating between these two conditions.

\section{Methods}

Subjects

Between October 2012 and August 2014, we prospectively performed standardized nerve ultrasound examinations in patients who suffered from MMN and ALS as ascertained by clinical and electrophysiological examinations as well as laboratory findings. In addition, we examined a healthy control group comprising medical staff or patients with other neurological diseases (such as epilepsy, headache, and vertigo), but without history of neuromuscular disorders and with unremarkable neurological examination. The study was registered in the German Clinical Trials Register 
(DRKS-ID: DRKS0005253) and approved by the local ethics committee (No. 3663-01/13 and EKZN 2014-230). Informed consent was obtained from all patients and controls. Inclusion criteria included diagnosis of ALS according to clinical signs and electromyography according to revised El-Escorial and Awaji criteria [4, 5], or diagnosis of MMN according to the EFNS criteria [29]. All patients underwent a clinical neurological examination, peripheral nerve ultrasound (PNUS), nerve conduction studies (NCS), and electromyography. Healthy controls underwent a clinical examination, PNUS and NCS.

\section{Ultrasound}

Ultrasonography was performed using a high frequency $14 \mathrm{MHz}$ probe real-time linear array scanner (ZONARE Ultrasound systems). Ultrasonography was performed bilaterally in different nerves of the upper and lower limbs and in the neck. Nerves were scanned in axial planes and the cross-sectional area (CSA) of each nerve was measured at standardized anatomical points as described before [11, $23]$; in short - the median nerve in the upper arm, before penetrating the pronator teres muscle (elbow) next to the brachial artery, and in the middle of the forearm, the ulnar nerve in the upper arm and at the mid-forearm; the tibial nerve in popliteal space (proximal) and at medial malleolus before its division into plantar nerves (ankle); the fibular nerve $2 \mathrm{~cm}$ above the fibular head, and the sural nerve between the lateral and medial head of the gastrocnemic muscle. In addition, the CSA of the vagus nerve in the carotid sheath beneath the carotid bifurcation and the longitudinal diameter of the $6^{\text {th }}$ cervical nerve root after leaving the processus transversus were measured. Measurement of nerves at entrapment sites (median nerve and ulnar nerve at the wrist, fibular nerve at the fibular head) was explicitly avoided. Nerve enlargement was defined according to reference and boundary values taken from the literature [11, 30]. Additionally, the maximum and minimum CSA of the median and ulnar nerve were evaluated by screening the whole nerve from the axilla to the wrist as recommended in the literature [31]. CSA was traced inside the hyperechoic rim of the nerve. Analysis of ultrasound data was performed both online and off-line. Approximately, $40 \mathrm{~min}$ were needed for a complete ultrasound examination of each patient. A second examiner evaluated all ultrasound measurements off-line once again. Both examiners were blinded to the electrophysiological measurements of the patients.

Nerve conduction studies and electromyography

Nerve conduction studies were performed in MMN and ALS patients as well as in controls at anatomical sites corresponding to the nerve ultrasound measurements using a standard electro-neurophysiologic device (Synergy 15.0, VIASYS Healthcare UK Ltd.). Measurements were carried out on the median nerve (from wrist up to the axilla and Erb point), the ulnar nerve (from wrist up to the axilla and Erb point), the tibial nerve, the fibular nerve, and the sural nerve. Compound motor action potential (CMAP), motor conduction velocity (CV), distal motor latency and F-wave response were measured in all sensorimotor nerves (median, ulnar, tibial, and fibular) as well as sensory nerve action potential (SNAP) and sensory conduction velocity (CV) in median, ulnar, and sural nerves. Conduction blocks were defined as at least a $50 \%$ reduction in the amplitude of the proximal CMAP compared to the amplitude of the distal CMAP and a $<30 \%$ increase of proximal to distal negative peak duration. In some MMN patients, high voltage motor-evoked potentials were performed. Electromyography was performed in cases of suggested ALS in all the defined four anatomical regions according to the revised El-Escorial criteria (bulbar, trunk, arms and legs, in detail: tongue, paravertebral thoracic, biceps brachii, abductor pollicis brevis, vastus lateralis and tibialis anterior).

\section{Statistics}

For statistical analysis, IBM SPSS Statistics, version 19 (Chicago, IL) was used. The unpaired $t$ test was used to evaluate differences concerning epidemiological data of the groups. One-way ANOVA was used to detect differences of nerve CSAs and diameter of 6th cervical nerve root between the groups (ALS, MMN, and controls), unpaired $t$ test was used to calculate differences between MMN and ALS, ALS and healthy controls, or MMN and controls. Post-hoc analysis was undertaken using Bonferroni correction due to multiple $t$ tests. Chi square test was used to compare the frequencies of nerve enlargement in the groups. Receiver operating characteristic (ROC) curve analysis was performed to evaluate the differences in the number of detected nerve enlargements between MMN and ALS and to define a cutoff value for differentiation between both pathologies with reliable sensitivity and specificity. The ROC curve plots for each threshold value, the proportion of false positives (i.e., 1 specificity on $\mathrm{X}$-axis) against the proportion of true positives (i.e., sensitivity on $y$-axis). The overall precision of the diagnosis is given by the area under the curve (AUC). An AUC of 1 represents highest accuracy, whereas an AUC of 0.5 represents purely random decisions independent from the measurements with no diagnostic value of the test. Intraclass correlation coefficients (ICC) were calculated to evaluate off-line inter-rater and intra-rater reliability for measurement of CSA and root diameter. Pearson 
correlation coefficients were calculated to quantify correlations between NCS and ultrasonic findings.

\section{Results}

A total of 25 patients and 28 healthy controls were included in the study. MMN was diagnosed in 8 patients according to the EFNS criteria [29], detection of conduction blocks in nerve conduction studies, and elevated anti-GM1-IgM antibodies and/or therapeutic response to intravenous immunoglobulins during an observational period of at least 6 months. Six patients were treated with immunoglobulins during the examination period, one received mycophenolatmofetil and one was therapy naive. 7 out of 8 patients with MMN had elevated anti-GM1-antibodies and/ or measureable response to different therapeutic regimes. 17 patients received a diagnosis of definite or probable ALS according to Awaji criteria and revised El-Escorial criteria [4, 5]. Table 1 gives an overview of baseline characteristics.

Table 2 shows NCS results of median and tibial nerves. The results of the other nerves are not shown herein, but were also used for diagnosis. In most of the MMN patients, ultrasound and electrophysiology were not carried out at the same time point. In seven MMN patients, at least one conduction block was detected according to EFNS criteria, mostly located in the median nerve of the forearm. In the ALS group, the mean CMAP amplitudes of the tibial and the median nerve, but not the ulnar and fibular nerve, were significantly reduced in comparison to the controls $(p<0.05)$. Neither signs of demyelination, e.g. reduced conduction velocity, nor conduction blocks were found in
ALS patients. Sensory nerve action potentials and nerve conduction velocity of the sural nerve revealed no significant differences between MMN and ALS (Fig. 1).

Table 3, Figs. 1 and 2 show the median CSA and/or diameter results as well as the box plots of all peripheral nerves, the vagus, and the $6^{\text {th }}$ cervical nerve root for each group. One-way ANOVA revealed significant differences for CSAs for all measurement points of the sensorimotor nerves, the vagus, and for the diameter of the 6th cervical nerve root, most prominent in the proximal part of the median nerve and the distal part of the tibial nerve $(p<0.001)$. The unpaired $t$ test with Bonferroni correction revealed significant differences for all these nerves in the MMN group compared to the ALS group and to healthy controls (excluding the vagus). However, no significant differences could be detected between ALS patients and controls (Table 3). No significant differences were found in the sural nerves between the different groups. Pathological nerve enlargement was defined according to published boundary values $[11,30]$. Figure 3 a shows the results of ROC curve analysis of the number of detected nerve enlargements to diagnose $\mathrm{MMN}$ in contrast to ALS. A number of $\geq 4$ nerve enlargements reveal a reliable boundary value with a sensitivity of $87.5 \%$ and a specificity of $>94 \%$ to differentiate MMN from ALS. In contrast, ROC curve analysis showed no diagnostic value for detection of nerve enlargements to differentiate between ALS and controls (Fig. 3b). In MMN patients, significantly more nerve enlargements (all peripheral nerves and the 6th cervical nerve root) could be detected (Fig. 3c, Chi square test, $p<0.001)$. The sural nerve was excluded from this comparison, because it has-as a pure sensory nerve-no impact on the differentiation of MMN and ALS.

Table 1 Baseline characteristics of the study population

\begin{tabular}{|c|c|c|c|c|}
\hline & Control & MMN & ALS & $p$ value \\
\hline$N$ & 28 & 8 & 17 & \\
\hline Mean age in years \pm SD & $52.8 \pm 17.2$ & $55.6 \pm 11.2$ & $65.6 \pm 13.3$ & 0.129 \\
\hline Gender: men:women & $17: 13$ & $8: 0$ & $9: 6$ & 0.063 \\
\hline Height in $\mathrm{cm} \pm \mathrm{SD}$ & $174.0 \pm 6.3$ & $179.5 \pm 10.7$ & $170.7 \pm 9.8$ & 0.100 \\
\hline Weight in $\mathrm{kg} \pm \mathrm{SD}$ & $68.0 \pm 18.1$ & $81.2 \pm 13.1$ & $71.4 \pm 21.1$ & 0.530 \\
\hline Duration of disease in months $\pm \mathrm{SD}$ & NA & $41.2 \pm 21.6$ & $18.8 \pm 18.3$ & 0.016 \\
\hline Therapy & & $\begin{array}{l}6 \text { with IvIg } \\
1 \text { with } \mathrm{MMF} \\
1 \text { no therapy }\end{array}$ & & \\
\hline Clinical onset & & $\begin{array}{l}7 \text { Arm } \\
1 \text { Leg }\end{array}$ & $\begin{array}{l}7 \text { Arm } \\
6 \text { Leg } \\
4 \text { bulbar onset }\end{array}$ & \\
\hline Others & & Anti-GM1-IgN & & \\
\hline
\end{tabular}

ALS amyotrophic lateral sclerosis, Anti-GMI-IgM-AB Anti-ganglioside M1 IgM antibodies, $\mathrm{cm}$ centimeter, IvIg intravenous immunoglobulin, $\mathrm{kg}$ kilogram, $M M F$ mycophenolatmofetil, $M M N$ multifocal motor neuropathy, $N A$ not available

Significant value is shown in bold 
Table 2 Nerve conduction studies

$A L S$ amyotrophic lateral sclerosis, $C V$ conduction velocity, $C M A P$ composed motor action potential, EFNS

European Federation of

Neurological Societies/

Peripheral Nerve Society, $M M N$ multifocal motor neuropathy, $S D$ standard deviation

Significance was set $p<0.05$. Significant values are shown in bold

\begin{tabular}{lllll}
\hline & Control & MMN & ALS & ANOVA \\
\hline Motor CV median nerve (m/s) & Mean 53.4 & Mean 38.0 & Mean 55.1 & $\boldsymbol{p}=\mathbf{0 . 0 0 2}$ \\
& SD 4.6 & SD 9.8 & SD 5.1 & \\
CMAP amplitude median nerve $(\mathrm{mV})$ distal & Mean 9.8 & Mean 5.7 & Mean 4.6 & $\boldsymbol{p}=\mathbf{0 . 0 4 0}$ \\
& SD 3.3 & SD 4.7 & SD 0.7 & \\
CMAP amplitude median nerve $(\mathrm{mV})$ proximal & Mean 9.3 & Mean 2.9 & Mean 4.3 & $\boldsymbol{p}=\mathbf{0 . 0 2 0}$ \\
& SD 3.0 & SD 4.7 & SD 4.0 & \\
Motor CV tibial nerve(m/s) & Mean 43.4 & Mean 38.1 & Mean 42.9 & $p=0.104$ \\
& SD 2.2 & SD 7.2 & SD 4.3 & \\
CMAP amplitude tibial nerve (mV) distal & Mean 14.5 & Mean 5.1 & Mean 6.7 & $\boldsymbol{p}=\mathbf{0 . 0 0 2}$ \\
& SD 5.8 & SD 2.2 & SD 6.7 & \\
CMAP amplitude tibial nerve (mV) proximal & Mean 12.7 & Mean 3.2 & Mean 5.1 & $\boldsymbol{p}=\mathbf{0 . 0 0 8}$ \\
& SD 5.5 & SD 2.6 & SD 4.7 & \\
Definite Conduction block(according to EFNS, 29) & None & 4 median & None & \\
& & 1 ulnar & & \\
& & 2 tibial & & \\
\hline
\end{tabular}

The intranerve CSA variability (ICSAV) was calculated for the median and the ulnar nerve (CSAmax/CSAmin). In MMN, the ICSAV of median nerve was significantly increased compared to healthy controls $(p<0.05)$, whereas in the ulnar nerve no significance was found $(p=0.08)$. In addition, no significant differences were found between ALS and MMN (see Table 3). Pearson correlation revealed no significant relationship between nerve conduction velocity and CSA measurements, between conduction block site and ultrasonic focal nerve enlargement in MMN and between amount of axonal damage as well as CMAP amplitude reduction and nerve CSA in ALS. Sub-group analysis (e.g. between bulbar onset and spinal onset ALS) was not performed due to small patient numbers. The intra-rater intraclass correlation coefficient (ICC) of the off-line nerve ultrasound measurements was 0.995 , and interrater ICC was 0.990 .

\section{Discussion}

Diagnostic differentiation between ALS and MMN presents a difficult clinical and electrophysiological challenge. Heterogeneity of clinical onset and time course often delays the diagnosis of ALS [3]. Moreover, a number of other diseases may imitate ALS-especially in the early stage of the disease. Some of the differential diagnoses may offer a different prognosis and implicate specific therapeutic options. A correct diagnosis of ALS is even difficult using electromyography. However, additional muscle ultrasound may facilitate diagnosis of ALS [6]. In $\mathrm{MMN}$, diagnosis can also be difficult due to the lack of detectable electrophysiological parameters such as conduction blocks [32]. Transcranial magnetic stimulation or triple-stimulation techniques can be helpful here, but are elaborate and not easily accessible [29]. Further, the diagnostic accuracy of anti-GM1 testing ranges between 30 and $80 \%$ [33].

Ultrasound of the peripheral nervous system proves to be a useful additional diagnostic tool for diagnosis of hereditary neuropathies [20, 21, 34-36] and acquired immune-mediated acute and chronic neuropathies [13-20, 37-40]. In MMN, focal nerve enlargement has already been previously described in ultrasound and magnetic resonance imaging studies [20, 24, 25, 38]. Focal nerve enlargements in MMN are not surprising as focal conduction blocks are found in several nerves and at different sites. In contrast, pronounced generalized or multifocal nerve enlargement has not been described in ALS to date. Several authors have even described a reduction of nerve CSA or nerve root diameter as a sign of atrophic nerves $[27,28]$. Therefore, it has been suggested that nerve atrophy is an expression of LMN loss.

In this study, in a population of patients with proven diagnosis of MMN and probable or definite diagnosis of ALS, ultrasonic measurements of the peripheral nerves revealed significant differences between both disorders. Median values of all peripheral sensorimotor nerves and the vagus nerve were significantly enlarged in MMN in comparison to ALS and healthy controls (Figs. 1, 2, 4). In ALS, no significant differences were found compared to healthy controls. However, the overall median CSA enlargement was not as pronounced as described in other populations, e.g. chronic inflammatory demyelinating polyradiculoneuropathy (CIDP) $[16,18,19]$. This may be due to the patchy and interindividual variable pattern of nerve 
Fig. 1 Box plots of the amplitude of sensory nerve action potentials of the sural nerve in $\mu \mathrm{V}$ and ultrasonic cross-sectional area measurements of the median and the ulnar nerve at different anatomical landmarks in $\mathrm{mm}^{2}$ in multifocal motor neuropathy (MMN), amyotrophic lateral sclerosis (ALS) and healthy controls. Significant results of the unpaired $t$ test with Bonferroni corrections between MMN and ALS and between MMN and controls are in bold print. No significances (ns) were observed between ALS and controls. Significance was set $p<0.05$. The nonsignificant $p$ values are marked with ns and are shown in Table 3 sural nerve SNAP amplitude

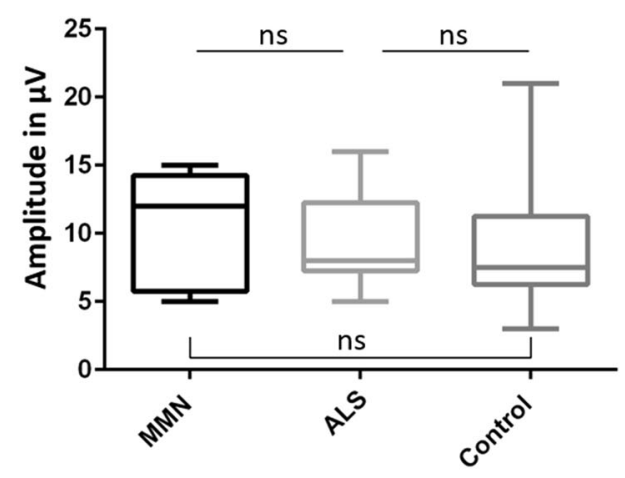

median nerve elbow

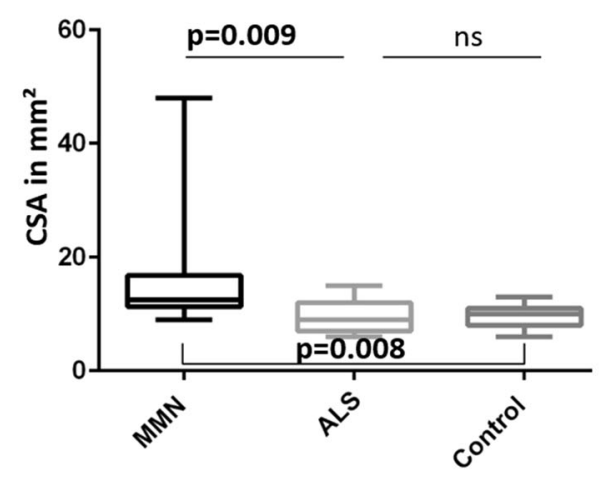

ulnar nerve upper arm

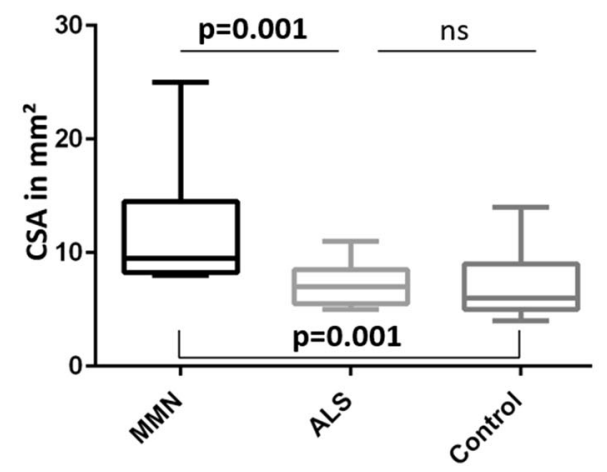

median nerve upper arm
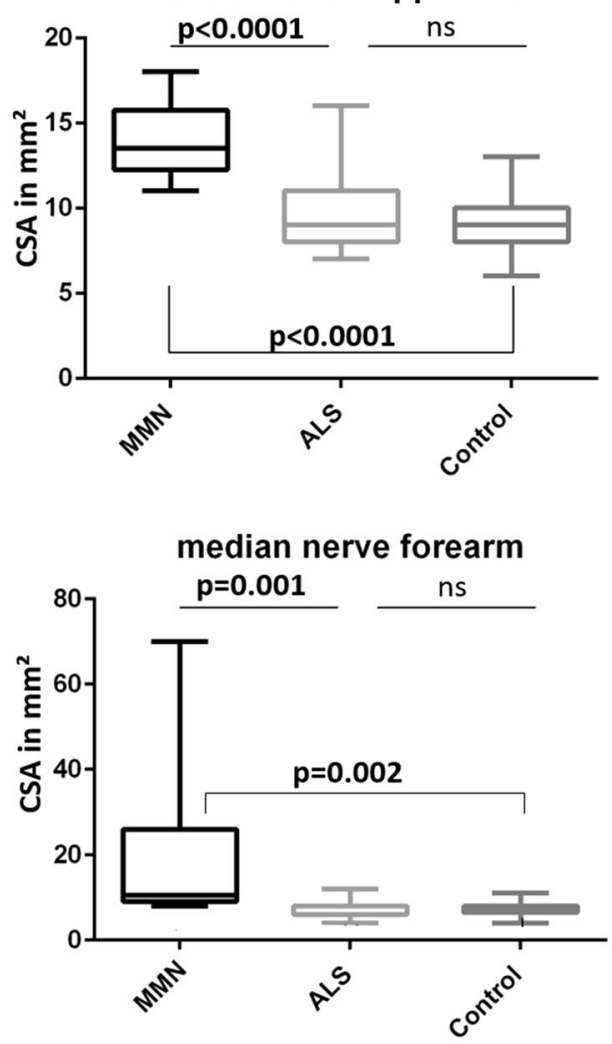

ulnar nerve forearm

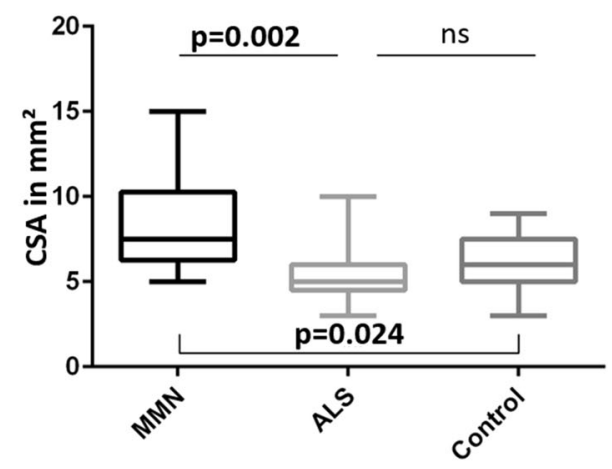

involvement in MMN. Examination of the sural nerves as purely sensory nerves showed no differences between ALS, MMN, and controls. Thus, these findings are in line with previous studies [24-28, 38].

The median diameter of the 6th cervical nerve root was significantly increased in MMN-probably due to focal inflammation of the nerve roots [38]. In contrast, no significant differences to the controls could be detected in ALS patients. In a later stage of ALS, nerve root atrophy may be detected due to LMN degeneration [27, 28]. However, we need to consider that we only analyzed the C6 nerve root diameter-due to the simplicity, which serves as a limitation. To further evaluate the use of cervical nerve root ultrasound, it is necessary to analyse more nerve roots at more anatomical sites as well as to measure the CSA instead of diameter.

The slight hypertrophy of the vagus in MMN patients is an interesting finding which needs to be discussed in more detail since MMN patients normally show no autonomic dysregulation. Vagus hypertrophy is often described in the Guillain-Barré syndrome (GBS) [39]. Nevertheless, patients with inherited neuropathies or CIDP variants also show vagus enlargement $[40,41]$ without autonomic dysregulation. All these neuropathies can also affect other cranial nerves [8, 40, 41]. In addition to its parasympathetic nerve fibers, the vagus nerve also contains motor fibers (i.e. 
Fig. 2 Box plots of the crosssectional area measurements of the fibular, the tibial, the sural and the vagal nerve in $\mathrm{mm}^{2}$, and the diameter of the cervical nerve root 6 in $\mathrm{mm}$ in multifocal motor neuropathy (MMN), amyotrophic lateral sclerosis (ALS) and healthy controls. Significant results of the unpaired $t$ test with Bonferroni corrections between MMN and ALS and between MMN and controls are in bold print. No significances (ns) were observed between ALS and controls. Significance was set $p<0.05$. The non-significant $p$ values are marked with ns and are shown in Table 3
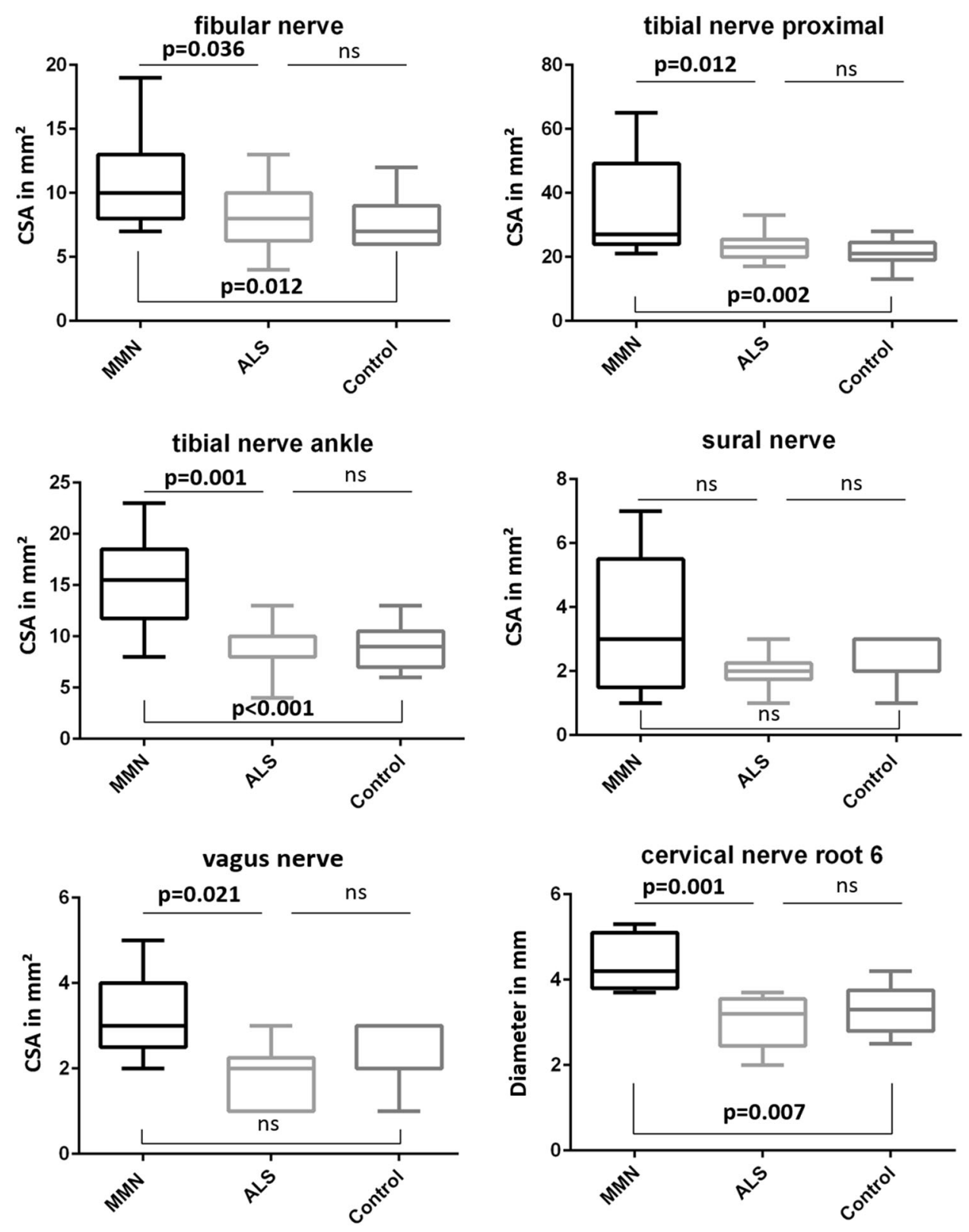

pharyngeal nerve and superior laryngeal nerve). Accordingly, vagus nerve hypertrophy in MMN may be caused by the involvement of these motor fibers and may be correlated to dysphagia or hoarseness rather than to autonomic dysregulation. In addition, it may be interesting to further analyse other cranial nerves, such as the phrenic nerve or the facial nerve in MMN using PNUS. However, the significance of this result has to be interpreted with care as in the current study, only two out of eight MMN patients showed an increased CSA of the vagus nerve over $3 \mathrm{~mm}^{2}$, a value that was not significant compared to the controls.

The most obvious difference between the groups was the significantly higher number of nerve enlargements in MMN in comparison to ALS and controls (Fig. 3c, $p<0.001)$. However, single nerve enlargement was also found in some ALS patients. Thus, to differentiate MMN from ALS with high sensitivity and specificity, boundary values for the number of required nerve pathologies are necessary. In our study population, ROC curve analysis revealed nerve enlargement in at least 4 out of 10 landmarks to serve as a reliable boundary value with a sensitivity of $87.5 \%$ and a specificity of $94.1 \%$ to differentiate MMN from ALS.

The ICSAV, described as a useful tool for differentiating between MMN and CIDP by means of evaluating focal or diffuse nerve enlargement (CSAmax/CSAmin; 31), showed significant differences in our MMN population in the median nerve in comparison to the control group 

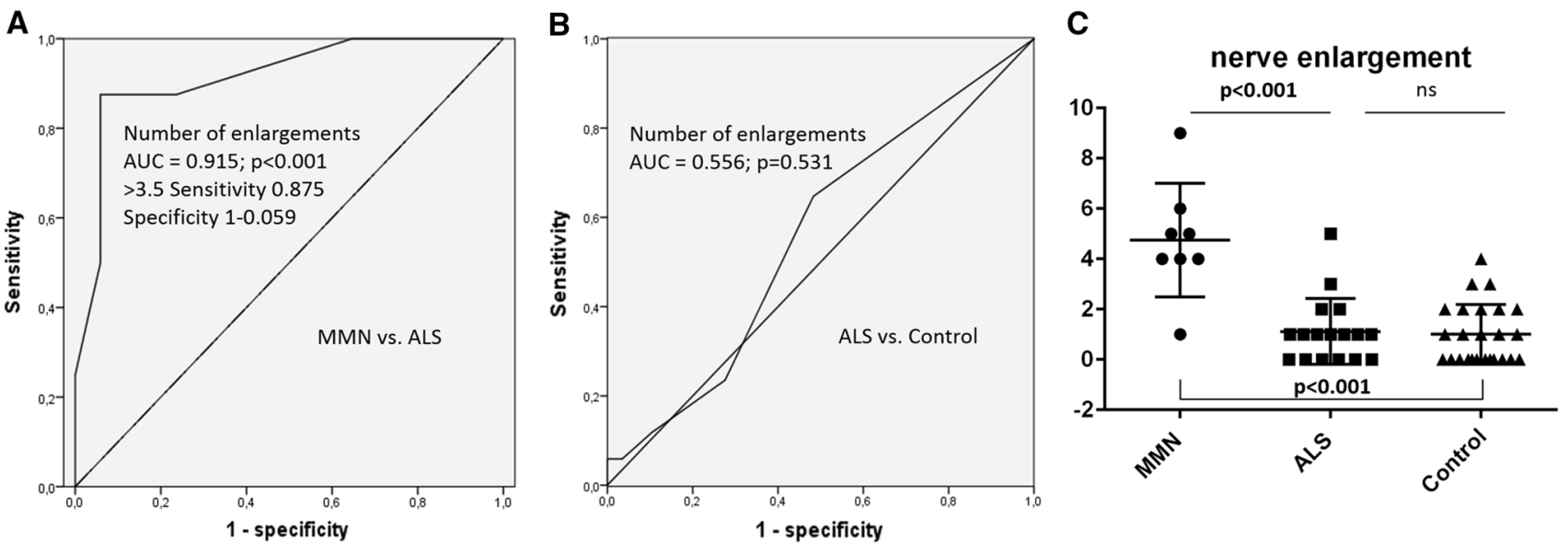

Fig. 3 a-c Receiver operating characteristics (ROC) curve of the number of nerve and/or cervical nerve root enlargements to differentiate between MMN and ALS (a) or between ALS and controls (b). The detection of nerve enlargement at 4 or more measurement points has a sensitivity of $87.5 \%$ and a specificity of $94.1 \%$ to diagnose MMN $(p<0.001)$. In contrast, no differences in the number of enlarged nerves were found between ALS and controls $(p=0.531)$.

c The scatter blots of the number of enlarged nerves in all patients and healthy controls. The Chi square test shows significant differences between MMN and ALS $(p<0.001)$ and between MMN and controls $(p<0.001)$, but not between ALS and controls $(p>0.05, n s$ non-significant). Significances are set $p<0.05$ and are shown in bold print

Table 3 Median Ultrasound results

\begin{tabular}{|c|c|c|c|c|c|c|c|}
\hline $\begin{array}{l}\text { Median CSA in } \\
\mathrm{mm}^{2} / \text { diameter } \\
\text { in } \mathrm{mm} \text { (range) }\end{array}$ & Control & MMN & ALS & ANOVA & $\begin{array}{l}t \text { test with } \\
\text { Bonferroni } \\
\text { MMN vs. ALS }\end{array}$ & $\begin{array}{l}t \text { test with } \\
\text { Bonferroni } \\
\text { ALS vs. } \\
\text { control }\end{array}$ & $\begin{array}{l}t \text { test with } \\
\text { Bonferroni } \\
\text { MMN vs. } \\
\text { control }\end{array}$ \\
\hline $\begin{array}{l}\text { Median nerve } \\
\text { upper arm }\end{array}$ & $9.0(6.0-13.0)$ & $13.5(11.0-18.0)$ & $9.0(7.0-16.0)$ & $p<0.0001$ & $p<0.0001$ & $p=0.405$ & $p<0.0001$ \\
\hline $\begin{array}{l}\text { Median nerve } \\
\text { elbow }\end{array}$ & $10.0(6.0-13.0)$ & $12.5(9.0-48.0)$ & $9.0(6.0-15.0)$ & $p=0.005$ & $p=0.009$ & $p=1.0$ & $p=0.008$ \\
\hline $\begin{array}{l}\text { Median nerve } \\
\text { forearm }\end{array}$ & $7.5(4.0-11.0)$ & $10.5(8.0-70.0)$ & $6.0(4.0-12.0)$ & $p=0.001$ & $p=0.001$ & $p=1.0$ & $p=0.002$ \\
\hline $\begin{array}{l}\text { Ulnar nerve upper } \\
\text { arm }\end{array}$ & $6.0(4.0-14.0)$ & $9.5(8.0-25.0)$ & $7.0(5.0-11.0)$ & $p=0.001$ & $p=0.001$ & $p=0.997$ & $p=0.001$ \\
\hline $\begin{array}{l}\text { Ulnar nerve } \\
\text { forearm }\end{array}$ & $6.0(3.0-9.0)$ & $7.5(5.0-15.0)$ & $5.0(3.0-10.0)$ & $p=0.003$ & $p=0.002$ & $p=0.599$ & $p=0.024$ \\
\hline $\begin{array}{l}\text { Tibial nerve } \\
\text { popliteal }\end{array}$ & $21.0(13.0-28.0)$ & $27.0(21.0-65.0)$ & $13.0(17.0-33.0)$ & $p=0.003$ & $p=0.012$ & $p=0.880$ & $p=0.002$ \\
\hline Tibial nerve ankle & $9.0(6.0-13.0)$ & $15.5(8.0-23.0)$ & $10.0(4.0-13.0)$ & $p<0.0001$ & $p=0.001$ & $p=1.0$ & $p<0.0001$ \\
\hline $\begin{array}{l}\text { Fibular nerve } \\
\text { popliteal }\end{array}$ & $7.0(6.0-12.0)$ & $10.0(7.0-19.0)$ & $8.0(4.0-13.0)$ & $p=0.013$ & $p=0.036$ & $p=1.0$ & $p=0.012$ \\
\hline Sural nerve calf & $2.0(1.0-3.0)$ & $3.0(1.0-7.0)$ & $2.0(1.0-3.0)$ & $p=0.083$ & $p=0.157$ & $p=1.0$ & $p=0.101$ \\
\hline $\begin{array}{l}\text { Vagus nerve } \\
\text { carotid sheath }\end{array}$ & $2.0(1.0-3.0)$ & $3.0(2.0-5.0)$ & $2.0(1.0-3.0)$ & $p=0.021$ & $p=0.020$ & $p=1.0$ & $p=0.069$ \\
\hline $\begin{array}{l}\text { C6 longitudinal } \\
\text { proc. transv. }\end{array}$ & $3.4(2.5-4.2)$ & $4.2(3.7-5.3)$ & $3.2(2.0-3.7)$ & $p=0.001$ & $p=0.001$ & $P=0.751$ & $p=0.007$ \\
\hline $\begin{array}{l}\text { ICSAV median } \\
\text { nerve }\end{array}$ & 1.38 SD 0.2 & 1.80 SD 0.7 & $1.58 \mathrm{SD} 0.4$ & $p=0.052$ & $p>0.05$ & $P>0.05$ & $p<0.05$ \\
\hline $\begin{array}{l}\text { ICSAV ulnar } \\
\text { nerve }\end{array}$ & $1.28 \mathrm{SD} 0.2$ & $1.48 \mathrm{SD} 0.3$ & $1.47 \mathrm{SD} 0.5$ & $p=0.213$ & $p>0.05$ & $p>0.05$ & $p>0.05$ \\
\hline
\end{tabular}

$C S A$ cross-sectional area, $A L S$ amyotrophic lateral sclerosis, ICSAV intranerve CSA variability, $M M N$ multifocal motor neuropathy Significance is set $p<0.05$. Significant differences are shown in bold 

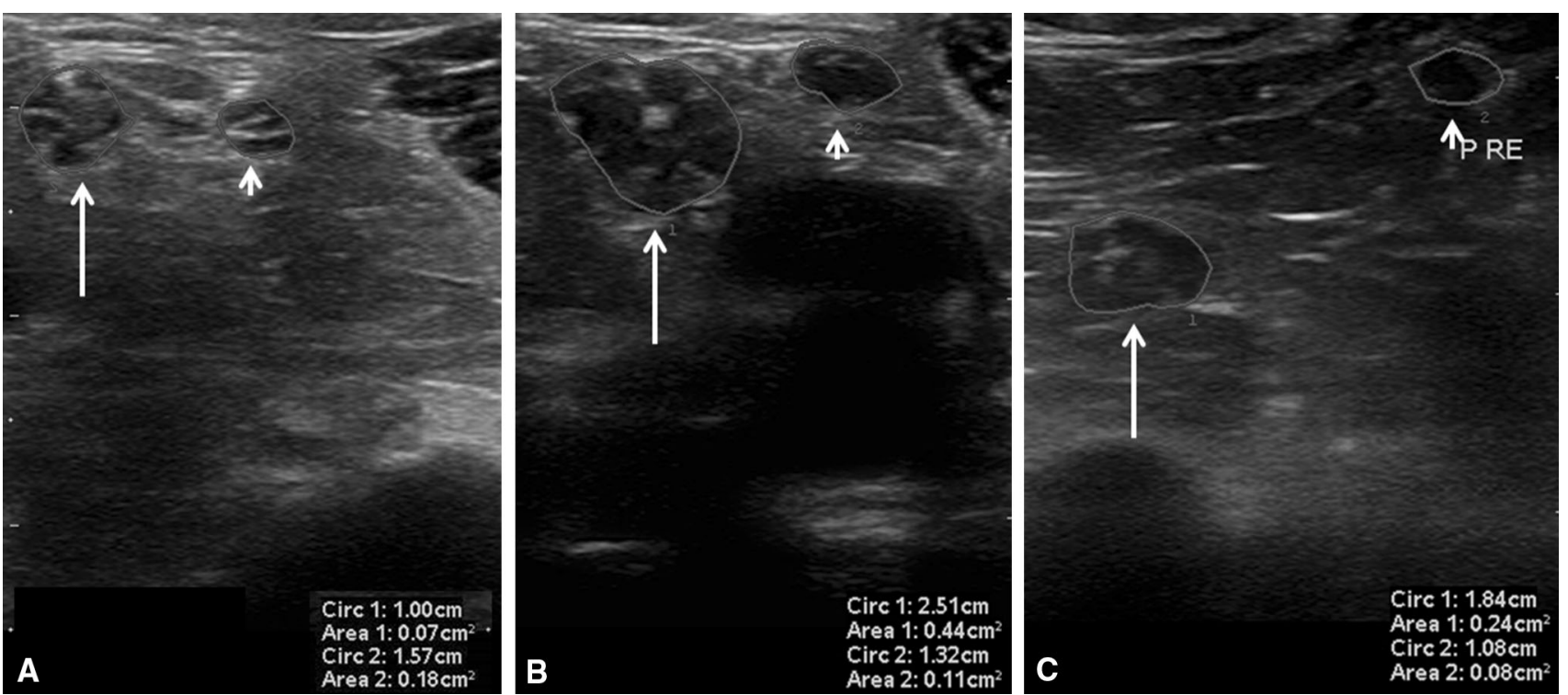

Fig. 4 Axial ultrasound of the tibial (long arrow) and fibular (short arrow) nerve in the poplitea in a patient with amyotrophic lateral sclerosis (a) and in a patient with multifocal motor neuropathy (b) compared to a healthy control (c). In multifocal motor neuropathy

$(p<0.05)$. However, ICSAV showed no significant differences between ALS and MMN, and as such does not represent a differentiation tool here. This could be explained by the relatively small CSA values in the median and ulnar nerve in ALS with a predilection for distal nerve segments, which may have influenced the variability score. However, significant differences in comparison to the controls were not found in our ALS group.

The pathophysiology of nerve enlargement in MMN is not yet clear. In chronic demyelinating neuropathies, reand demyelination (onion bulbs) may play a role, while in acute and subacute neuropathies, focal edema and inflammation seem to cause nerve enlargement [39]. In addition, the time course of nerve enlargement and nerve atrophy in both diseases remains quite unclear so far. In CIDP, several changes of nerve CSA, echogenicity and cervical nerve root diameter depending on disease duration have been described [18].

Nerve enlargement was observed in nerves with and without $\mathrm{CB}$ in this study. The finding describing a missing correlation between nerve enlargement and NCS pathology has been reported several times in immune-mediated neuropathies as well as in MMN [16, 24, 25]. Hence, we suggest that NCS and PNUS characterize different pathologies with regard to nerve damage. Ultrasound provides focal information reflecting morphology whereas NCS offers information indicating functionality of the nerve. However, it has to be considered that this study focussed on nerve ultrasound, and ultrasonic examinations have been done more detailed than NCS. cross-sectional area (CSA) is increased (tibial nerve: $44 \mathrm{~mm}^{2}$ in MMN, $18 \mathrm{~mm}^{2}$ in ALS, $24 \mathrm{~mm}^{2}$ in control; fibular nerve: $11 \mathrm{~mm}^{2}$ in MMN, $7 \mathrm{~mm}^{2}$ in ALS, $8 \mathrm{~mm}^{2}$ in control)

With respect to study limitations, we note that the study population was small, the disease duration was significantly longer in the MMN group, and the men to women ratio predominated in the MMN group. All of the above factors could have had an influence on the observed differences in both groups. Other baseline characteristics showed slight but not significant differences such as age, height and weight and their influence on nerve morphology also remains unclear [30]. In addition, the time points of electrophysiological testing and ultrasound examination differed in some of the patients. Thus, a comparison of nerve pathology in PNUS and in NCS has some bias potential and data need to be interpreted with care. The sensitivity and specificity of this method were evaluated in a study population in which this method was developed and thus should be confirmed in a larger population in a multicentre, controlled study. A further challenge will be the differentiation of $\mathrm{MMN}$ and other neuropathies using PNUS.

The next step should be the examination of therapy naive patients in the beginning stages of their disease to have the same conditions in different groups and to evaluate the value of PNUS as diagnostic tool in the beginning of the diseases.

In conclusion, systematic ultrasonic measurements of CSA in different peripheral nerves and the vagus nerve, and measuring the diameter of the cervical nerve roots provide an additional tool for differentiating between ALS and MMN with ROC curve-based boundaries for the detected number of nerve enlargements. In the majority of 
cases, both diagnoses can be undertaken by performing a meticulous clinical examination and electrodiagnostic studies. However, diagnosis can be difficult in the early stage of the disease due to lack of signs of upper motoneuron in ALS [3] and because of missing conduction blocks in MMN [32]. Thus, the ultrasonic finding of multifocal nerve enlargement can strengthen the diagnosis of MMN in contrast to ALS, and therefore, warrants a beneficial therapy with immunoglobulins.

Acknowledgments The research was supported by the German Center for Sepsis Control and Care (CSCC, funded by the Ministry of Education and Research (BMBF), Grant No 01 E0 1002. The authors thank Nasim Kroegel for her help in language editing.

Conflicts of interest The authors declare that they have no conflict of interest.

Ethical standards The study was registered in the German Clinical Trials Register (DRKS-ID: DRKS0005253) and approved by the local ethics committee (No. 3663-01/13 and EKZN 2014-230). Informed consent was obtained from all patients and controls.

\section{References}

1. Brooks BR (1994) El Escorial World Federation of Neurology criteria for the diagnosis of amyotrophic lateral sclerosis. Subcommittee on Motor Neuron Diseases/Amyotrophic Lateral Sclerosis of the World Federation of Neurology Research Group on Neuromuscular Diseases and the El Escorial "Clinical limits of amyotrophic lateral sclerosis" workshop contributors. J Neurol Sci 124(Suppl):96-107

2. Hardiman O, van den Berg LH, Kiernan MC (2011) Clinical diagnosis and management of amyotrophic lateral sclerosis. Nat Rev Neurol 7:639-649

3. Kraemer M, Buerger M, Berlit P (2010) Diagnostic problems and delay of diagnosis inn amyotrophic lateral sclerosis. Clin Neurol Neurosurg 112:103-105

4. Brooks BR, Miller RG, Swash M et al (2000) El Escorial revisited: revised criteria for the diagnosis of amyotrophic lateral sclerosis. Amyotroph Lateral Scler. 1:293-299

5. de Carvalho M, Dengler R, Eisen A et al (2008) Electrodiagnostic criteria for diagnosis of ALS. Clin Neurophysiol 119:497-503

6. Grimm A, Prell T, Décard BF, et al. (2014) Muscle ultrasonography as an additional diagnostic tool for the diagnosis of amyotrophic lateral sclerosis. Clin Neurophysiol. doi:10.1016/j. clinph.2014.06.052 [Epub ahead of print]

7. Pestronk A, Cornblath DR, Ilyas AA et al (1988) A treatable multifocal motor neuropathy with antibodies to GM1ganglioside. Ann Neurol 24:73-78

8. Nobile-Orazio E (2001) Multifocal motor neuropathy. J Neuroimmunol 115:4-18

9. Cornblath DR, Sumner AJ, Daube J et al (1991) Conduction block in clinical practice. Muscle Nerv 14:869-871

10. Nobile-Orazio E, Gallia F (2013) Multifocal motor neuropathy: current therapies and novel strategies. Drugs 73(5):397-406

11. Grimm A, Heiling B, Schumacher U et al (2014) Ultrasound differentiation of axonal and demyelinating neuropathies. Muscle Nerv 50:976-983

12. Hobson-Webb LD (2013) Neuromuscular ultrasound in polyneuropathies and motor neuron disease. Muscle Nerv 47:790-804
13. Kerasnoudis A (2013) Nerve ultrasound in a case of chronic inflammatory demyelinating neuropathy. Muscle Nerv 47:443-446

14. Kerasnoudis A, Pitarokoili K, Behrendt V et al (2013) Correlation of nerve ultrasound, electrophysiological, and clinical findings in post Guillain-Barré syndrome. J Peripher Nerv Syst 18:232-240

15. Kerasnoudis A, Pitarokoili K, Behrendt V et al (2013) Cross sectional reference values for sonography of peripheral nerves and brachial plexus. Clin Neurophysiol 124:1881-1888

16. Kerasnoudis A, Pitarokoili K, Behrendt V, et al. (2014) Correlation of nerve ultrasound, electrophysiological and clinical findings in chronic inflammatory demyelinating polyneuropathy. J Neuroimaging. doi:10.1111/jon.12079 [Epub ahead of print]

17. Matsuda M, Ikeda S, Sakurai S et al (1996) Hypertrophic neuritis due to chronic inflammatory demyelinating polyradiculoneuropathy (CIDP): a postmortem pathological study. Muscle Nerv 19:163-169

18. Padua L, Granata G, Sabatelli M et al (2014) Heterogeneity of root and nerve ultrasound pattern in CIDP patients. Clin Neurophysiol 125:160-165

19. Zaidman CM, Al-Lozi M, Pestronk A (2009) Peripheral nerve size in normal and patients with polyneuropathy: an ultrasound study. Muscle Nerv 40:960-966

20. Zaidman CM, Harms MB, Pestronk A (2013) Ultrasound of inherited vs. acquired demyelinating polyneuropathies. J Neurol 260:3115-3121

21. Schreiber S, Oldag A, Kornblum C et al (2013) Sonography of the median nerve in CMT1A, CMT2A, CMTX, and HNPP. Muscle Nerv 47:385-395

22. Ito T, Kijima M, Watanabe T et al (2007) Ultrasonography of the tibial nerve in vasculitic neuropathy. Muscle Nerve 35:379-382

23. Grimm A, Décard BF, Bischof A, et al. (2014) Ultrasound of the peripheral nerves in systemic vasculitic neuropathies. J Neurol Sci. doi:10.1016/j.jns.2014.09.017 [Epub ahead of print]

24. Kerasnoudis A, Pitarokoili K, Behrendt V, et al. (2014) Multifocal motor neuropathy: correlation of nerve ultrasound, electrophysiological and clinical findings. J Peripher Nerv Syst 19:165-174

25. Beekman R, van den Berg LH, Franssen H et al (2005) Ultrasonography shows extensive nerve enlargements in multifocal motor neuropathy. Neurology 65:305-307

26. Cartwright MS, Walker FO, Griffin LP et al (2011) Peripheral nerve and muscle ultrasound in amyotrophic lateral sclerosis. Muscle Nerve 44:346-351

27. Nodera H, Takamatsu N, Shimatani Y et al (2014) Thinning of cervical nerve roots and peripheral nerves in ALS as measured by sonography. Clin Neurophysiol 125:1906-1911

28. Schreiber S, Abdulla S, Debska-Vielhaber G, et al. (2014) Peripheral nerve ultrasound in ALS phenotypes. Muscle Nerve. doi:10.1002/mus.24431 [Epub ahead of print]

29. Joint Task Force of the EFNS and the PNS (2010) European Federation of Neurological Societies/Peripheral Nerve Society guideline on management of multifocal motor neuropathy. Report of a joint task force of the European Federation of Neurological Societies and the Peripheral Nerve Society-first revision. J Peripher Nerv Syst 15(4):295-301

30. Boehm J, Scheidl E, Bereczki D et al (2014) High-resolution ultrasonography of peripheral nerves: measurements on 14 nerve segments in 56 healthy subjects and reliability assessments. Ultraschall Med 35:459-467

31. Padua L, Martinoli C, Pazzaglia C et al (2012) Intra- and internerve cross-sectional area variability: new ultrasound measures. Muscle Nerve 45:730-733

32. Nodera H, Bostock H, Izumi Y et al (2006) Activity-dependent conduction block in multifocal motor neuropathy: magnetic fatigue test. Neurology 67:280-287 
33. Van Schaik IN, Bossuyt PMM, Brand A et al (1995) The diagnostic value of GM1 antibodies in motor neuron disorders and neuropathies: a meta-analysis. Neurology 45:1570-1577

34. Hooper DR, Lawson W, Smith L et al (2011) Sonographic features in hereditary neuropathy with liability to pressure palsies. Muscle Nerve 44:862-867

35. Beekman R, Visser LH (2002) Sonographic detection of diffuse peripheral nerve enlargement in hereditary neuropathy with liability to pressure palsies. J Clin Ultrasound 30:433-436

36. Martinoli C, Schenone A, Bianchi S et al (2002) Sonography of the median nerve in Charcot- Marie-Tooth disease. AJR Am J Roentgenol 178:1553-1556

37. Scheidl E, Böhm J, Simó M et al (2012) Ultrasonography of MADSAM neuropathy: focal nerve enlargements at sites of existing and resolved conduction blocks. Neuromuscul Disord $22: 627-631$
38. Van Es HW, Van den Berg LH, Franssen H et al (1997) Magnetic resonance imaging of the brachial plexus in patients with multifocal motor neuropathy. Neurology 48:1218-1224

39. Grimm A, Décard BF, Axer H (2014) Ultrasonography of the peripheral nervous system in the early stage of Guillain-Barré syndrome. J Peripher Nerv Syst. doi:10.1111/jns.12091

40. Grimm A, Thomaser AL, Peters N, et al. (2014) Vagal hypertrophy in immune-mediated neuropathy visualised with highresolution ultrasound (HR-US). J Neurol Neurosurg Psychiatry. doi:10.1136/jnnp-2014-308271 [Epub ahead of print]

41. Cartwright MS, Brown ME, Eulitt $P$ et al (2009) Diagnostic nerve ultrasound in Charcot-Marie-Tooth disease type 1B. Muscle Nerv 40:98-102 